\title{
Efectos de diferentes prácticas de preparación del terreno y fertilización sobre el crecimiento inicial del Pinus spp en el NE argentino*
}

Effects of different site preparation and fertilization methods on growth of Pinus spp in the NE of Argentina

\author{
R. FERNANDEZ ${ }^{1}$, F. RODRIGUEZ ASPILLAGA ${ }^{2}$, A. LUPI ${ }^{1}$, A. HERNANDEZ ${ }^{2}$, H. REIS ${ }^{1}$ \\ ${ }^{1}$ EEA Montecarlo INTA. Av El Libertador 2472 (3384) Montecarlo. Argentina. Formronte@inta.gov.ar. \\ Fac. Ciencias Forestales, UNaM. Bertoni 124 (3380). Eldorado, Misiones, Argentina. \\ ${ }^{2}$ Productos Tissue S.A. Posadas. Misiones. Argentina.
}

\begin{abstract}
SUMMARY
Growth results of two experiments, obtained six months after plantation are presented. The objective was to evaluate the response of Pinus taeda L. and Pinus elliottii E. under different silvicultural treatments in Corrientes, northeastern Argentina. Response of Pinus taeda, applying 0, 22.5 and $45 \mathrm{~g} \mathrm{~N} \mathrm{pl}^{-1}$; 0, 45 and $90 \mathrm{~g} \mathrm{P}_{2} \mathrm{O}_{5} \mathrm{pl}^{-1}$; also 0,36 and $72 \mathrm{~g} \mathrm{~K}_{2} \mathrm{O} \mathrm{pl}{ }^{-1}$, on Kandiudalf soil was evaluated. Statistical differences were observed between N, P and $\mathrm{NxP}$ interaction. $\mathrm{P}$ doses had a positive effect, while $\mathrm{N}$ doses presented a negative effect. Response of Pinus taeda and Pinus elliottii on deficient drainage sites prepared with disk ploughs or mounding with applications of NPK 15$30-12$, at 0,100 and $200 \mathrm{~g} \mathrm{pl}^{-1}$ to both species was also evaluated. The best growth results were obtained when both species were planted on moundings. A positive trend was detected in Pinus taeda when fertilizer was applied.
\end{abstract}

Key words: silviculture, Pinus taeda L., Pinus elliottii E., soil mounding fertilization.

\section{RESUMEN}

Se presentan los resultados de crecimiento obtenidos, a los seis meses de efectuada la plantación, cuyos objetivos son evaluar la respuesta de Pinus taeda L. y de Pinus elliottii E. frente a diversas prácticas de establecimiento en el noreste de la provincia de Corrientes, Argentina. Sobre un Kandiudalf se evaluó la respuesta del Pinus taeda a la aplicación de $0,22.5$ y $45 \mathrm{~g} \mathrm{de} \mathrm{N} \mathrm{pl}^{-1}$; 0,45 y $90 \mathrm{~g} \mathrm{P}_{2} \mathrm{O}_{5} \mathrm{pl}^{-1}$ y 0,36 y $72 \mathrm{~g} \mathrm{~K}_{2} \mathrm{O} \mathrm{pl}^{-1}$. Se observaron diferencias estadísticas entre las distintas dosis de $\mathrm{N}$, de $\mathrm{P}$ y la interacción de $\mathrm{NxK}$. La aplicación de fósforo se tradujo en un efecto positivo, mientras que la aplicación de nitrógeno muestra una tendencia inversa. También se evaluó la respuesta del Pinus taeda y Pinus elliottii, implantados en sitios con drenaje deficiente preparados mediante rastra y camellones, en los cuales fueron aplicados, además, 0, 100 y $200 \mathrm{~g} \mathrm{pl}^{-1}$ de NPK 15-30-12. Los mayores crecimientos se presentaron cuando ambas especies fueron implantadas en camellones. Para el Pinus taeda se detectó una tendencia positiva ante la aplicación del fertilizante.

Palabras claves: silvicultura, Pinus taeda L., Pinus elliottii E., fertilización, camellones.

\section{INTRODUCCION}

La actividad forestal, en particular la referida a los bosques implantados, manifiesta en estos últimos años interesantes y continuos incrementos en productividad, tanto a nivel silvícola como industrial (Fernández 1997). En este sentido en el nordeste argentino, particularmente en el norte de la provincia de Corrientes, se están incorporando a esta actividad tierras tradicionalmente dedicadas

* Trabajo presentado en X Silvotecna. IUFRO Conference. Site Productivity Improvement. 
al uso agrícola-ganadero. Esta expansión apunta también a la incorporación de tierras bajas, consideradas marginales, generalmente por condiciones de anegamiento temporario.

Nambiar (1998) sostiene que las estrategias para el establecimiento de plantaciones envuelven combinaciones de diversas técnicas, entre ellas las de preparación física del suelo y la fertilización; y que la selección de una determinada combinación surge de considerar factores tales como características edáficas, topografía, requerimientos y tolerancia de la especie, el clima y los costos.

La construcción de camellones es una práctica utilizada en áreas anegables cuando se busca crear un ambiente adecuado para el desarrollo radicular ya que mejora la aireación y la disponibilidad de nutrientes. Boden (1984) observó que el crecimiento del Pinus elliottii en sitios preparados con camellones se tradujo en incremento del volumen total del orden del $20-40 \%$ respecto del alcanzado en lotes sin esta forma de preparación.

Según Snowdon y Waring (1984) la fertilización al establecimiento de las plantaciones puede incrementar la productividad mediante la reducción del período necesario para alcanzar la máxima producción y/o a través de incrementos en el nivel de máxima producción del rodal.

Toro (1995) indica que la aplicación de fertilizantes al momento de la plantación, tanto en $P$. radiata como en Eucalyptus spp, es una práctica habitual en numerosos países, mediante la cual puede incrementarse significativamente el crecimiento inicial. Según datos del Programa Cooperativo de Nutrición Forestal de la Universidad de Carolina del Norte, durante el año 1995 fueron fertilizadas al inicio de la plantación cerca de 38.000 ha de $P$. taeda y $P$. elliottii, sólo en el sudeste de los EE.UU.

En Argentina, no es común la fertilización en el establecimiento de las plantaciones. No obstante, se cuenta con resultados a nivel operacional y, en algunos casos, con apoyo de ensayos, los cuales indican que, en determinados sitios, esta práctica puede asociarse con incrementos en el crecimiento, tanto en Eucalyptus grandis (Dalla Tea 1995) como en Pinus taeda.

Esta contribución tiene como objeto presentar los resultados iniciales de experiencias que se desarrollan con el apoyo financiero del Proyecto Forestal de Desarrollo-S.A.G.PyA.-BIRF, en acuerdo entre el Instituto Nacional de Tecnología
Agropecuaria (INTA) y la empresa Productos Tissue S.A. (PROTISA).

Estas experiencias tienen como objetivo:

- Evaluar la respuesta del Pinus taeda a la aplicación de distintas dosis de N, P y K en suelos rojos del NE de Comentes.

- Evaluar la respuesta a diferentes técnicas de preparación del terreno para el cultivo del Pinus taeda y Pinus elliottii, en suelos de drenaje deficiente del NE de Corrientes.

\section{RESPUESTA DEL PINUS TAEDA A LA APLICACION DE DISTINTAS DOSIS DE N, P Y K EN SUELOS ROJOS DEL NE DE CORRIENTES}

\section{MATERIAL Y METODOS}

La experiencia se localiza en el NE de la provincia de Corrientes, Argentina, en el Campo Timbaúva, propiedad de la empresa Productos Tissue S.A, a $\operatorname{los} 56^{\circ}$ de longitud oeste y $28^{\circ}$ de latitud sur, próxima a la localidad de Gobernador Virasoro. La región se caracteriza por una temperatura media anual del orden de $\operatorname{los} 21^{\circ} \mathrm{C}$, con un régimen de lluvias uniforme de $1.700 \mathrm{~mm}$ anuales. El ensayo se ubica sobre una loma de suelo rojo profundo, arcilloso, bien drenado; cuyas características químicas se presentan en el cuadro 1 . Taxonómicamente pertenece al Gran Grupo de los Kandiudalfes (INTA, 1990). El relieve es del tipo suave ondulado y la pendiente media es del 3-5\%. Proviene de un uso agrícola-ganadero.

La preparación del terreno para la plantación se realizó mediante labranza vertical con subsolador hasta $\operatorname{los} 70-80 \mathrm{~cm}$ de profundidad, luego se aplicó sobre la línea una rastra y un rotovator de 1.3 $m$ de ancho a los fines de preparar la cama de plantación y permitir además la aplicación de preemergentes. Los plantines utilizados en el ensayo corresponden a Pinus taeda L origen Marión, de procedencia Florida, U.S.A., los cuales fueron producidos en contenedores de $93 \mathrm{~cm}^{3}$. Al momento de llevarlas a campo se seleccionaron aquellas que presentaron un diámetro de cuello de 3-4 mm y una altura de 25-30 cm. En agosto de 1997 se realizó la plantación a un distanciamiento de $4 \mathrm{~m}$ entre líneas y $1.75 \mathrm{~m}$ en la línea. Los primeros días de septiembre se aplicaron las distintas dosis de fertilizante simulando una aplicación operacio- 


\section{CUADRO 1}

Características químicas del suelo.

Chemical soil characteristics

\begin{tabular}{|c|c|c|c|c|c|c|c|c|c|c|}
\hline Espesor & $\begin{array}{c}\mathrm{pH} \\
\mathrm{H}_{2} \mathrm{O}\end{array}$ & $\begin{array}{c}\mathrm{pH} \\
\mathrm{KCl}\end{array}$ & $\mathrm{MO}$ & $\mathrm{N}$ & $\mathrm{P}$ & $\mathrm{Ca}^{2+}$ & $\mathrm{Mg}^{2+}$ & $\mathrm{K}^{+}$ & $\begin{array}{c}\text { Acidez } \\
\text { Interc. }\end{array}$ & $\begin{array}{c}\mathrm{Al}^{3+} \\
\text { Interc. }\end{array}$ \\
\hline$(\mathrm{cm})$ & \multicolumn{2}{|c|}{} & \multicolumn{2}{|c|}{$\%$} & $\mathrm{ppm}$ & \multicolumn{5}{|c|}{$\mathrm{Cmol} / \mathrm{kg}$} \\
\hline $0-10$ & 4.5 & 3.8 & 6.7 & 0.40 & 2.3 & 4.9 & 3.3 & 0.10 & 3.8 & 2.6 \\
$10-30$ & 5.0 & 4.1 & 4.9 & 0.35 & 1.3 & 5.2 & 3.2 & 0.04 & 3.3 & 2.4 \\
$30-60$ & 4.7 & 3.8 & 1.4 & 0.01 & 0.3 & 10.2 & 6.4 & 0.01 & 1.5 & 0.9 \\
\hline
\end{tabular}

nal, esto es, aplicando los fertilizantes en forma de dos chorrillos paralelos de $30 \mathrm{~cm}$ de longitud, a cada lado de la planta, a una distancia de $15 \mathrm{~cm}$ desde del tallo, e incorporándolos al suelo a una profundidad de $8 \mathrm{~cm}$. Entre los cuidados culturales posplantación se encuentran el control de hormigas y el control de malezas en toda la superficie por un período de 24 meses.

El diseño utilizado corresponde a un factorial de $3^{3}$, dispuesto en bloques completos al azar con tres repeticiones. Los tratamientos son combinaciones de tres niveles de N, P y K aplicados en forma de urea (45-46\% de $\mathrm{N}$ ), superfosfato triple $\left(46 \% \mathrm{P}_{2} \mathrm{O}_{5}\right)$ y cloruro de potasio $\left(60 \%\right.$ de $\mathrm{K}_{2} \mathrm{O}$ ). Para cada nutriente, los distintos niveles a evaluar son: nitrógeno: $0,22.5$ y $45 \mathrm{~g} \mathrm{~N} \mathrm{pl}^{-1}$ (corresponde a 0,50 y $100 \mathrm{~g}$ de urea por planta); fósforo: 0, 45 y $90 \mathrm{~g} \mathrm{P}_{2} \mathrm{O}_{5} \mathrm{pl}^{-1}$ (corresponde a 0, 98 y $196 \mathrm{~g}$ de superfosfato triple por planta) y potasio: 0, $36 \mathrm{y}$ $72 \mathrm{~g} \mathrm{~K}_{2} \mathrm{O} \mathrm{pl}{ }^{-1}$ (corresponde a 0, 60 y $120 \mathrm{~g}$ cloruro de potasio por planta). Cada parcela se compone de 56 plantas, de las cuales sólo 30 conforman la parcela de medición, correspondiendo el resto a bordura.

El análisis de los datos se efectuó mediante el análisis de varianza y el test de comparación de medias de Tukey.

En febrero de 1998 se realizó el primer relevamiento de altura total (Ht) y diámetro al nivel de cuello (DAC). A partir de estos datos se procedió al cálculo del factor de productividad (FP) según la siguiente ecuación: $\mathrm{FP}=\mathrm{DAC}^{2} \mathrm{x} \mathrm{Ht}$.

\section{RESULTADOS Y DISCUSION}

El análisis estadístico (cuadro 2) realizado respecto del comportamiento de la altura total (Ht), del DAC y del factor de productividad (FP) detectó diferencias altamente significativas, al 1\%, para las dosis de $\mathrm{N}$, P y la interacción $\mathrm{NxP}$; mientras que la interacción $\mathrm{NxK}$ sólo mostró diferencias para el DAC al 5\%. No se observaron diferencias al analizar la aplicación de K, PxK y NxPxK.

\section{CUADRO 2}

Efecto de la aplicación de distintas dosis de $\mathrm{N}$ y $\mathrm{P}$ sobre el crecimiento inicial de Pinus taeda Marion,

a los 6 meses desde la plantación.

Effect of different levels of $\mathrm{N}$ and $\mathrm{P}$ on Pinus taeda Marion growth, six months after planting.

\begin{tabular}{|c|c|c|c|c|c|c|}
\hline & \multicolumn{3}{|c|}{$\begin{array}{c}\text { Dosis de } N \\
\left(\mathrm{~g} \cdot \mathrm{pl}^{-1}\right)\end{array}$} & \multicolumn{3}{|c|}{$\begin{array}{c}\text { Dosis de } \mathrm{P}_{2} \mathrm{O}_{5} \\
\left(\mathrm{~g} \cdot \mathrm{pl}^{-1}\right)\end{array}$} \\
\hline & 0 & 22.5 & 45 & 0 & 45 & 90 \\
\hline Ht $(\mathrm{cm})$ & $40.47^{\mathrm{a}}$ & $37.94^{b}$ & $35.33^{\mathrm{c}}$ & $35.23^{\mathrm{c}}$ & $38.22^{b}$ & $40.29^{\mathrm{a}}$ \\
\hline DAC $(\mathrm{mm})$ & $7.43^{\mathrm{a}}$ & $7.20^{\mathrm{a}}$ & $6.65^{b}$ & $6.36^{\mathrm{c}}$ & $7.25^{b}$ & $7.68^{\mathrm{a}}$ \\
\hline $\mathrm{FP}\left(\mathrm{cm}^{3}\right)$ & $22.78^{a}$ & $20,64^{d}$ & $15.92^{b}$ & $14.41^{\mathrm{c}}$ & $20.61^{b}$ & $24.32^{d}$ \\
\hline
\end{tabular}

Letras diferentes indican diferencias estadísticas según Tukey con un nivel de significancia de 0.05 .

Ht: altura total; DAC: diámetro a la altura del cuello; FP: Factor de productividad. 
Tal como puede observarse en el cuadro 2, la aplicación de nitrógeno se asoció a una disminución del crecimiento del pino manifestado tanto por medio de la altura total como el diámetro del cuello. Tendencias similares fueron obtenidas para diferentes especies del género Pinus por Costa Muniz et al. (1975), Solberg (1975) y Fernández et al. (1995). Particularmente los primeros indican en su discusión una posible interferencia del $\mathrm{N}$ en la absorción de P. Además, Fowells y Kraus, citados por Costa Muniz et al. (1975), indican que la aplicación de altas dosis de $\mathrm{N}$ puede inhibir la formación de micorrizas en plantas de Pinus taeda.

En cuanto al $\mathrm{P}$, los datos manifiestan un incremento en el crecimiento con el aumento de las dosis aplicadas. Resultados similares, aunque a edades más avanzadas, fueron obtenidos por Binkley (1993), Buford y McKee (1986) y Haywood y Burton (1990) al evaluar el efecto de la fertilización inicial sobre el crecimiento en diámetro a la altura del pecho y volumen medio del Pinus taeda.

Respecto de la interacción NxP (cuadro 3), se observa que el efecto positivo del $\mathrm{P}$, tanto para la altura, el DAC y el factor de productividad, es más pronunciado ante la ausencia de nitrógeno o bien, cuando se aplican las dosis media y baja de este último. Ryden et al. (1977) citados por Ballard (1984) indican que la aplicación de fósforo, conjuntamente con el nitrógeno, tiene frecuentemente un efecto sinérgico sobre la utilización del primero. Sin embargo en este trabajo se detectó un comportamiento aparentemente antagónico ya que, independientemente de la dosis de fósforo aplicada, se observó una tendencia hacia la disminución del crecimiento a medida que se incrementó la dosis de nitrógeno. Estos resultados son coincidentes con los obtenidos por Buford y McKee (1986), quienes indican que la pérdida de crecimiento puede deberse a un incremento en la competencia de los microorganismos por el fósforo a consecuencia de un incremento poblacional como respuesta a una mayor oferta de nitrógeno.

Respecto de la interacción NxK, tal como se ha indicado anteriormente, sólo el DAC manifestó diferencias estadísticas al 5\% (cuadro 4), lo cual podría indicar que, en edades tempranas, esta variable puede manifestarse más sensible como respuesta a esta interacción. Similar a lo discutido para $\mathrm{NxP}$, en este caso también los mayores crecimientos se observaron en aquellos tratamientos que recibieron potasio en ausencia de nitrógeno. Particularmente, la aplicación de la dosis máxima de nitrógeno se tradujo en crecimientos, inclusive inferiores al testigo sin fertilizar $\left(\mathrm{N}_{1} \mathrm{~K}_{1}\right)$.

Finalmente, al evaluar la combinación NPK, los mejores crecimientos se manifestaron en los tratamientos $\mathrm{N}_{2} \mathrm{P}_{3} \mathrm{~K}_{3}(8.3 \mathrm{~mm}$ de DAC, $43.2 \mathrm{~cm} \mathrm{de}$ altura y $31.8 \mathrm{~cm}^{3}$ de FP), $\mathrm{N}_{1} \mathrm{P}_{3} \mathrm{~K}_{3}(8.2 \mathrm{~mm}$ de DAC, $43.4 \mathrm{~cm}$ de altura y $\left.29.1 \mathrm{c} \mathrm{m}^{3} \mathrm{de} F P\right)$ y $\mathrm{N}_{1} \mathrm{P}_{2} \mathrm{~K}_{3}(8.1$ $\mathrm{mm}$ de DAC, $42 \mathrm{~cm}$ de altura y $26.2 \mathrm{~cm}^{3}$ de FP). Estos resultaron significativamente superiores al testigo sin fertilizar $\mathrm{N}_{1} \mathrm{P}_{1} \mathrm{~K}_{1}(6.5 \mathrm{~mm}$ de DAC, $37.8 \mathrm{~cm}$ de altura y $15.8 \mathrm{~cm}^{3}$ de FP). Los menores crecimientos se asociaron a tratamientos que no incluyeron al fósforo, tales como $\mathrm{N}_{2} \mathrm{P}_{1} \mathrm{~K}_{3}(5.9 \mathrm{~mm}$ de DAC, $31.7 \mathrm{~cm}$ de altura y $11.2 \mathrm{~cm}^{3}$ de FP), $\mathrm{N}_{3} \mathrm{P}_{1} \mathrm{~K}_{3} \quad(5.9 \mathrm{~mm}$ de DAC, $32.9 \mathrm{~cm}$ de altura $\mathrm{y}$ $11.6 \mathrm{~cm}^{3}$ de FP) y $\mathrm{N}_{2} \mathrm{P}_{1} \mathrm{~K}_{2}(6.2 \mathrm{~mm}$ de DAC, 34.7 $\mathrm{cm}$ de altura y $13.4 \mathrm{~cm}^{3}$ de FP).

\section{CUADRO 3}

Efecto de la interacción del $\mathrm{NxP}$ sobre el crecimiento en altura, diámetro de cuello y factor de productividad de Pinus taeda Marion, a los 6 meses desde la plantación.

Effect of NxP interaction on height, root collar diameter and productivity rate growth of Pinus taeda Marion, six months after planting.

\begin{tabular}{|c|c|c|c|c|c|c|c|c|c|}
\hline & \multicolumn{3}{|c|}{$\begin{array}{c}\text { Ht } \\
(\mathrm{cm})\end{array}$} & \multicolumn{3}{c|}{$\begin{array}{c}\text { DAC } \\
(\mathrm{mm})\end{array}$} \\
\hline & $\mathrm{N}_{1}$ & $\mathrm{~N}_{2}$ & $\mathrm{~N}_{3}$ & $\mathrm{~N}_{1}$ & $\mathrm{~N}_{2}$ & $\mathrm{~N}_{3}$ & $\mathrm{~N}_{1}$ & $\mathrm{~N}_{2}$ & $\mathrm{~N}$ \\
\hline $\mathrm{P}_{1}$ & $37.93^{\mathrm{b}}$ & $33.81^{\mathrm{c}}$ & $33.95^{\mathrm{c}}$ & $6.67^{\mathrm{d}}$ & $6.17^{\mathrm{e}}$ & $6.25^{\mathrm{de}}$ & $16.97^{\mathrm{cd}}$ & $12.95^{\mathrm{e}}$ & $13.31^{\mathrm{e}}$ \\
$\mathrm{P}_{2}$ & $41.74^{\mathrm{a}}$ & $38.17^{\mathrm{b}}$ & $34.80^{\mathrm{c}}$ & $7.79^{\mathrm{ab}}$ & $7.41^{\mathrm{bc}}$ & $6.53^{\mathrm{de}}$ & $25.44^{\mathrm{a}}$ & $21.42^{\mathrm{b}}$ & $14.97^{\mathrm{de}}$ \\
$\mathrm{P}_{3}$ & $41.74^{\mathrm{a}}$ & $41.89^{\mathrm{a}}$ & $37.24^{\mathrm{b}}$ & $7.82^{\mathrm{ab}}$ & $8.03^{\mathrm{a}}$ & $7.19^{\mathrm{c}}$ & $25.92^{\mathrm{a}}$ & $27.55^{\mathrm{a}}$ & $19.48^{\mathrm{bc}}$ \\
\hline
\end{tabular}

Letras diferentes indican diferencias estadísticas según Tukey con un nivel de significancia de 0.05.

Ref : Ht: Altura total. DAC- Diámetro a la altura del cuello. FP: Factor de productividad.

$\mathrm{N}_{1}: 0$ g N.pl ${ }^{-1} ; \mathrm{N}_{2}: 22.5 \mathrm{~g} \mathrm{~N} \cdot \mathrm{pl}^{-1}$ y $\mathrm{N}_{3}: 45 \mathrm{~g} \mathrm{~N} \cdot \mathrm{pl}^{-1} \cdot \mathrm{P}_{1}: \mathrm{Og} \mathrm{P}_{2} \cdot \mathrm{pl}^{-1} ; \mathrm{P}_{2}: 45 \mathrm{~g} \mathrm{P}_{2} \mathrm{O}_{5} \cdot \mathrm{pl}^{-1}$ y $\mathrm{P}_{3}: 90 \mathrm{~g} \mathrm{P}_{2} \mathrm{O}_{5} \cdot \mathrm{pl}^{-1}$ 


\section{CUADRO 4}

Efecto de la interacción del NxK sobre el crecimiento en altura, diámetro de cuello y factor de productividad de Pinus taeda Marion, a los 6 meses desde la plantación.

Effect of NxK interaction on height, root collar diameter and productivity rate growth of Pinus taeda Marion, six months after planting.

\begin{tabular}{|c|c|c|c|c|c|c|c|c|c|}
\hline & \multicolumn{3}{|c|}{$\begin{array}{c}\mathrm{Ht} \\
(\mathrm{cm})\end{array}$} & \multicolumn{3}{|c|}{$\begin{array}{l}\text { DAC } \\
(\mathrm{mm})\end{array}$} & \multicolumn{3}{|c|}{$\begin{array}{c}\mathrm{FP} \\
\left(\mathrm{cm}^{3}\right)\end{array}$} \\
\hline & $\mathrm{N}_{1}$ & $\mathrm{~N}_{2}$ & $\mathrm{~N}_{3}$ & $\mathrm{~N}_{1}$ & $\mathrm{~N}_{2}$ & $\mathrm{~N}_{3}$ & $\mathrm{~N}_{1}$ & $\mathrm{~N}_{2}$ & $\mathrm{~N}_{3}$ \\
\hline $\mathrm{K}_{1}$ & 39.69 & 38.51 & 36 & $7.1^{b c}$ & $7.1^{b c}$ & $6.9^{\mathrm{cd}}$ & 20.52 & 20.70 & 17.59 \\
\hline $\mathrm{K}_{2}$ & 40.51 & 37.72 & 36.27 & $7.4^{\mathrm{ab}}$ & $7.2^{b c}$ & $6.6^{\mathrm{de}}$ & 22.90 & 20.13 & 16.35 \\
\hline $\mathrm{K}_{3}$ & 41.22 & 37.60 & 33.71 & $7.7^{\mathrm{a}}$ & $7.2^{b c}$ & $6.3^{\mathrm{e}}$ & 24.92 & 21.08 & 13.82 \\
\hline
\end{tabular}

Letras diferentes indican diferencias estadísticas según Tukey con un nivel de significancia de 0.05. Ref.: $\mathrm{N}_{1}: 0 \mathrm{~g} \mathrm{~N} \cdot \mathrm{pl}^{-1} ; \mathrm{N}_{2}: 22.5 \mathrm{~g} \mathrm{~N} \cdot \mathrm{pl}^{-1}$ y $\mathrm{N}_{3}: 45 \mathrm{~g} \mathrm{~N} \cdot \mathrm{pl}^{-1} \cdot \mathrm{K}_{1}: 0 \mathrm{~g} \mathrm{~K}_{2} 0 \cdot \mathrm{pl}^{-1} ; \mathrm{K}_{2}: 36 \mathrm{~g} \mathrm{~K}, 0 . \mathrm{pl}^{-1}$ y $\mathrm{K}_{3}: 72 \mathrm{~g} \mathrm{~K}_{2} \mathrm{O} \cdot \mathrm{pl}^{-1}$. Ht: Altura total. DAC: Diámetro a la altura del cuello. FP: Factor de productividad.

\section{CONCLUSIONES}

Los resultados obtenidos hasta los 6 meses de efectuada la plantación indican que la aplicación de 45 y $90 \mathrm{~g} \mathrm{P}_{2} \mathrm{O}_{5} \mathrm{pl}^{-1}$ se tradujo en un efecto positivo sobre el crecimiento del Pinus taeda, expresado tanto en altura total como diámetro del cuello, mientras que la aplicación de 22.5 y $45 \mathrm{~g}$ $\mathrm{N} \mathrm{pl}^{-1}$ se asoció con la disminución de los referidos parámetros de crecimiento. Por su lado, se observó un comportamiento indiferente respecto de la aplicación de 36 y 72 g $\mathrm{K}_{2} \mathrm{O} \quad \mathrm{pl}^{-1}$.

\section{TECNICAS DE PREPARACION DEL TERRENO PARA EL CULTIVO DEL PINUS TAEDA Y PINUS ELLIOTTII EN SUELOS DE DRENAJE DEFICIENTE DEL NE DE CORRIENTES}

\section{MATERIAL Y METODOS}

La experiencia se localiza en el NE de la provincia de Corrientes, Argentina, en el Campo Pariopá, propiedad de la empresa Productos Tissue S.A. a $\operatorname{los} 56^{\circ}$ de longitud oeste y $28^{\circ}$ de latitud sur, próxima a la localidad de Santo Tomé.

La región se caracteriza por una temperatura media anual del orden de $\operatorname{los} 21^{\circ} \mathrm{C}$, con un régimen de lluvias uniforme y $1.700 \mathrm{~mm}$ anuales.

El ensayo se ubica en un área cuyo relieve es del tipo subnormal, ocupando una posición topográfica de bajo, con escurrimiento lento (Clase de drenaje 1 a 2; Etchevehere 1976). Estas áreas generalmente no se encuentran bajo uso y se conocen en la región como "tendido bajo". El suelo pertenece al Gran Grupo de los Haplacueptes (INTA 1990). y sus características químicas se presentan en el cuadro 5.

En noviembre de 1996 se procedió a aplicar los tratamientos de preparación del terreno de manera tal que particularmente para los camellones, intermediara un período de estabilización hasta el momento de la plantación. En agosto de 1997 se realizó la plantación a un distanciamiento de $4 \mathrm{~m}$ entre líneas y de $1.75 \mathrm{~m}$ en la línea. Los plantines de Pinus taeda utilizados en el ensayo fueron producidos en contenedores de $93 \mathrm{~cm}^{3}$ y al momento de llevarlos a campo se seleccionaron aquellos que presentaron un diámetro de cuello de 3-4 mm y una altura de 25-30 cm. Respecto de las parcelas de Pinus elliottii, inicialmente fueron utilizados plantines a raíz desnuda, pero debido al elevado porcentaje de fallas fue necesario proceder a su replante total a los 30 días de la plantación original, esta vez con mudas producidas en contenedores. La fertilización se realizó a los 30 días de la plantación. Entre los cuidados culturales posplantación se encuentran el control de hormigas y el control químico de malezas en toda la superficie por un período de 24 meses.

La experiencia se dispone bajo un diseño factorial de $2 \times 2 \times 3$. en bloques completos al azar con cuatro repeticiones. 


\section{CUADRO 5}

Características químicas del suelo.

Chemical soil characteristics

\begin{tabular}{|c|c|c|c|c|c|c|c|c|c|c|c|}
\hline Espesor & $\begin{array}{c}\mathrm{pH} \\
\mathrm{H}_{2} \mathrm{O}\end{array}$ & $\begin{array}{c}\mathrm{pH} \\
\mathrm{KCl}\end{array}$ & $\mathrm{MO}$ & $\mathrm{N}$ & $\mathrm{P}$ & $\mathrm{Ca}^{2+}$ & $\mathrm{Mg}^{2+}$ & $\mathrm{K}^{+}$ & $\begin{array}{c}\text { Acidez } \\
\text { Interc. }\end{array}$ & $\begin{array}{c}\mathrm{Al}^{3+} \\
\text { Interc. }\end{array}$ \\
\hline (cm) & \multicolumn{2}{|c|}{} & \multicolumn{2}{|c|}{$\%$} & $\mathrm{ppm}$ & \multicolumn{5}{|c|}{ Cmol/kg } \\
\hline $0-10$ & 4.7 & 4.0 & 3.9 & 0.18 & 1.9 & 3.9 & 2.2 & 0.11 & 1.5 & 1.1 \\
$10-30$ & 4.7 & 4.0 & 3.1 & 0.15 & 1.0 & 3.1 & 2.1 & 0.04 & 2.0 & 1.6 \\
$30-60$ & 4.7 & 3.9 & 2.5 & 0.14 & 0.9 & 2.3 & 2.5 & 0.01 & 2.5 & 1.9 \\
\hline
\end{tabular}

Entre los factores bajo evaluación se encuentran:

- Especies: se evalúa el crecimiento del Pinus taeda L y $P$. elliottii E.

- Técnicas de preparación del terreno:

- Rastra: comprende el aplastado de la vegetación herbácea, aplicación de quema y pasada de rastra.

- Camellón: camellones o "mounding" de 1.8 $\mathrm{m}$ de ancho y $40 \mathrm{~cm}$ de altura respecto del nivel original del terreno, luego de su estabilización.

- Niveles de fertilización: se comparan tres dosis de fertilizante compuesto NPK 15-30-12 en dosis de 0,100 y $200 \mathrm{~g} \mathrm{pl}^{-1}$.
El cuadro 6 presenta los tratamientos que surgen de la combinación de estos factores.

Cada parcela consta de 95 plantas y cubre una superficie de $532 \mathrm{~m}^{2}$. Las parcelas de medición fueron conformadas con 51 plantas, separadas entre sí por una línea de bordura, la que en el caso de parcelas de distintas especies se realizó con Pinus taeda.

El análisis de los datos se efectuó mediante el análisis de varianza y el test de comparación de medias de Tukey.

En febrero de 1998 se efectuó el primer relevamiento de altura total $(\mathrm{Ht})$ y diámetro al nivel de cuello (DAC). A partir de estos datos se procedió al cálculo del factor de productividad (FP) según la siguiente ecuación: $\mathrm{FP}=\mathrm{DAC}^{2} \mathrm{x} \mathrm{Ht}$.

\section{CUADRO 6}

Síntesis de tratamientos según factores a evaluar.

Synthesis of treatments according to factors on evaluation

\begin{tabular}{|c|c|c|c|}
\hline Especies & Preparación del terreno & Dosis de $15-30-12\left(\mathrm{~g} \cdot \mathrm{pl}^{-1}\right)$ & Tratamiento \\
\hline \multirow[t]{2}{*}{ Pinus taeda } & Camellón & $\begin{array}{r}0 \\
100 \\
200\end{array}$ & $\begin{array}{l}1 \\
2 \\
3\end{array}$ \\
\hline & Rastra & $\begin{array}{r}0 \\
100 \\
200\end{array}$ & $\begin{array}{l}4 \\
5 \\
6\end{array}$ \\
\hline \multirow[t]{2}{*}{ Pinus elliottii } & Camellón & $\begin{array}{r}0 \\
100 \\
200\end{array}$ & $\begin{array}{l}7 \\
8 \\
9\end{array}$ \\
\hline & Rastra & $\begin{array}{r}0 \\
100 \\
200\end{array}$ & $\begin{array}{l}10 \\
11 \\
12\end{array}$ \\
\hline
\end{tabular}




\section{RESULTADOS Y DISCUSION}

En el cuadro 7 se presentan los valores medios de diámetro del cuello, altura y factor de productividad alcanzados a los seis meses de efectuada la plantación, correspondientes a los diferentes tratamientos.

Al evaluar el comportamiento de las especies, pudo observarse que el Pinus Taeda manifestó crecimientos significativamente superiores al Pinus elliottii. Tal es así que el crecimiento en altura y diámetro a nivel del cuello fue 1.5 veces superior, mientras que para el caso del factor de productividad las diferencias se triplicaron. Sin embargo, debe tenerse en cuenta que el Pinus elliottii debió ser replantado y que su período de crecimiento en campo es 30 días menor que el de Pinus taeda. En cuanto a la sobrevivencia, manifestó un comportamiento similar al presentado por las variables de crecimiento, alcanzando valores medios del $82 \%$ para el Pinus taeda y $66 \%$ para Pinus elliottii.

Respecto de las dos técnicas de preparación del terreno evaluadas, el análisis de varianza mostró diferencias altamente significativas, tanto para la altura, el diámetro a nivel del cuello, como para el factor de productividad. La altura media del Pinus taeda y Pinus elliottii implantados en camellones fue $43.8 \mathrm{~cm}$, mientras que en sitios preparados con rastra alcanzaron una altura de $26.8 \mathrm{~cm}$. En cuanto al diámetro a nivel del cuello, los valores fueron de $7.2 \mathrm{~mm}$ y $5.1 \mathrm{~mm}$ respectivamente, mientras que para el factor de productividad las diferencias se presentan marcadamente superiores ya que el crecimiento de ambas especies implantadas en camellones triplica al ocurrido en sitios rastreados. En cambio, al analizar el efecto de las prácticas de preparación del terreno sobre la sobrevivencia, no se manifestaron diferencias estadísticas, alcanzando valores de 77 y $71 \%$ de sobrevivencia cuando las especies fueron implantadas en sitios preparados con camellones y rastra, respectivamente.

La figura 1 muestra las medias de los parámetros de crecimiento, a los seis meses desde la plantación, correspondientes a ambas especies, en función de la modalidad de preparación del terreno. Tal como se observa, ambas especies manifestaron mayores crecimientos cuando fueron implantadas en sistemas de camellones.

Respecto de la adición de nutrientes pudo observarse una respuesta positiva a la aplicación de fertilizante, pero sólo en las parcelas de Pinus taeda implantado en camellones (cuadro 7). Merece destacarse que en octubre y parte de noviembre de 1997 la región soportó precipitaciones superiores a las medias, consecuencia de lo cual el sitio del ensayo presentó condiciones de anegamiento. Debido a ello podría haberse producido un efecto de dilución del fertilizante, especialmente en los sitios en que el relieve no fue modificado (preparación con rastra).

\section{CUADRO 7}

Crecimiento en altura $(\mathrm{cm})$, diámetro del cuello $(\mathrm{mm})$, factor de productividad $\left(\mathrm{cm}^{3}\right)$ y sobrevivencia $(\%)$ de $P$. taeda y $P$. elliottii según modalidad de preparación del terreno y dosis de fertilizante.

Height $(\mathrm{cm})$, root collar diameter $(\mathrm{mm})$, productivity rate growth $\left(\mathrm{cm}^{3}\right)$ and survival $(\%)$ of $P$. taeda and $P$. elliottii according to site preparation methods and fertilizer doses.

\begin{tabular}{|l|c|c|c|c|c|c|c|c|c|c|c|c|}
\hline \multicolumn{1}{|c|}{} & \multicolumn{9}{c|}{ Pinus taeda } & \multicolumn{3}{c|}{ Pinus elliottii } \\
\cline { 2 - 13 } & \multicolumn{3}{|c|}{ Camellón } & \multicolumn{3}{c|}{ Rastra } & \multicolumn{3}{c|}{ Camellón } & \multicolumn{3}{c|}{ Rastra } \\
\cline { 2 - 13 } & 0 & 100 & 200 & 0 & 100 & 200 & 0 & 100 & 200 & 0 & 100 & 200 \\
Ht & 50.6 & 51.2 & 59.3 & 31.2 & 33.5 & 32.7 & 32 & 34.6 & 35.3 & 19.9 & 20.4 & 23.5 \\
DAC & 7.9 & 8.1 & 10.2 & 5.7 & 5.7 & 5.6 & 5.9 & 6.1 & 6.1 & 4.6 & 4.6 & 4.8 \\
FP & 34 & 37 & 67 & 10 & 12 & 10 & 11 & 14 & 13 & 4 & 4 & 5 \\
Sobrv. & 82.5 & 78 & 77 & 94 & 80 & 80 & 77 & 74.5 & 73 & 55 & 55 & 65 \\
\hline
\end{tabular}

Ref.: 0, 100 y 200 corresponde a los g/planta aplicados de 15-30-12, Ht: altura total (cm), DAC: Diámetro del cuello (mm), FP: Factor de Productividad $\left(\mathrm{cm}^{3}\right)$, Sobrev.: sobrevivencia $(\%)$. 


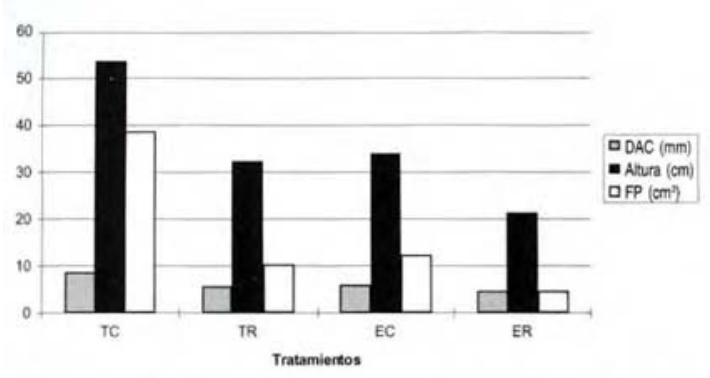

TC: $P$. taeda implantado en camellón. $\quad$ TR: $P$, taeda implantado en suelos rastreados. EC: $P$, elliottii implantado en camellón. ER: $P$. elliottii implantado en suelos rastreados.

Figura 1: Crecimiento de Pinus taeda y Pinus elliottii implantados en suelos con drenaje restringido, en función de la preparación del terreno.

Growth of Pinus taeda and Pinus elliottii in restricted drainage lands, in relation to site preparation methods.

Al evaluar la interacción especies-prácticas de preparación del terreno se observó que el porcentaje de sobrevivencia presentado por las parcelas de Pinus elliottii preparadas con rastra (58\%) resultó el menor de todos, y fue estadísticamente significativo respecto de las demás combinaciones. También se observaron diferencias significativas en la interacción Pinus taeda implantado con rastra ( $85 \%)$, respecto del Pinus elliottii sobre camellones $(75 \%)$. Finalmente, la sobrevivencia no se vio significativamente afectada al evaluar al Pinus taeda en sus diferentes sistemas de preparación (79 y $85 \%$ para camellones y rastra respectivamente) o bien, al comparar ambas especies implantadas en camellones (79 y 75\%) para Pinus taeda y Pinus elliottii respectivamente.

Resultados similares a los presentados en este trabajo fueron obtenidos por Pritchett y Smith (1974) citados por Pritchett (1986) sobre un Albaquult típico, donde observaron que a los 9 años el crecimiento en altura del Pinus taeda manifestó incrementos superiores al $90 \%$ respecto de sitios no preparados con camellones. Además, al evaluar el efecto de la aplicación del fertilizante, indican que si bien el mejor crecimiento en los camellones se debe en parte a una mejor nutrición, un beneficio importante provenía de un mejor drenaje. Por su lado Haines et al. (1975), citados por Pritchett (1986), informaron del efecto positivo de los camellones sobre el crecimiento a mediano plazo del Pinus elliottii, y que parcelas de esta especie presentaron a los 8.5 años una altura promedio superior en $1.8 \mathrm{~m}$ respecto de otras preparadas con rastra. Además, se indica que este beneficio puede disminuir a medida que los árboles avanzan en edad y, consecuentemente, su sistema radical explora volúmenes edáficos más profundos.

\section{CONCLUSIONES}

El análisis de los resultados correspondientes a los seis meses de efectuada la plantación indican que el crecimiento en altura y diámetro del cuello alcanzados en terrenos preparados con camellones, tanto para Pinus taeda como para Pinus elliottii, fueron significativamente mayores que los correspondientes a terrenos preparados con rastra. La altura media en áreas de camellones fue $43.8 \mathrm{~cm}$ contra $26.8 \mathrm{~cm}$ en sitios preparados con rastra. En cuanto al diámetro a nivel del cuello, los valores fueron de $7.2 \mathrm{~mm}$ y $5.1 \mathrm{~mm}$, respectivamente.

El Pinus taeda implantado sobre camellones manifestó respuestas positivas a la adición de fertilizante NPK.

\section{BIBLIOGRAFIA}

BALLARD, R. 1984. Fertilization of plantation. In: Chapter 12. Nutrition of Plantation Forest. London. $1^{\text {a }}$ ed., Academic Press Inc., 505 p.

BINKLEY, D., D. RICHTER, M.B. DAVID, B. CALDWEL. 1992. "Soil chemistry in a loblolly/longleaf Pine forest with interval burning", Ecological Applications 2 (2): 157-164.

BODEN, D. I. 1984. Early responses to different methods of site preparation for three commercial tree species. En: Proceedings Symposium on Site and Productivity of Fast Growing Plantations. IUFRO. Pretoria, South Africa.

BUFORD, M. A., W. H. MCKEE. 1986. Effects of site preparation, fertilization and genotype on loblolly pine growth and stand structure. Results at age 15. En: Proceedings of the Fourth Biennial Southern Silvicultural Research Conference. Atlanta, EE.UU., p. 378-383.

COSTA MUNIZ, P., G. BALDANZI, S. NETTO. 1975. "Ensaio de adubacao em Pinus elliotii e Pinus taeda no sul do Brasil", Floresta VI (1): 5-13.

DALLA TEA, F. 1995. Fertilización con dosis variables de P en Eucalyptus grandis. En: Informe PROFOME. Ensayos de experimentación adaptativa e investigación 1992-1994. Informe actividades 1994. INTA. Centros Regionales Misiones, Corrientes y Entre Ríos.

DONALD, D. G. M., P.W. LANGE, C.J. SCHUTZ, A.R. MORRIS. 1984. The application of fertilizers to pines in Southern Africa. En: Proceedings Symposium on Site and Productivity of Fast Growing Plantations. IUFRO. Pretoria, South Africa.

ETCHEVEHERE, P. 1976. Normas de reconocimiento de suelos. Publicación 152. INTA-Castelar. 2 Ed. Buenos Aires, Argentina, $211 \mathrm{p}$.

FERNANDEZ, R. A. 1997. "Hacia una producción forestal sostenible", SAGPyA Forestal 3:10-14. Buenos Aires. Argentina. 
FERNANDEZ, R. A., J. FAHLER, N. PAHR, A.M. LUPI, 1995. Respuesta del Pinus taeda a la aplicación de distintas dosis de N y P. En: Informe PROFOME. Ensayos de experimentación adaptativa e investigación 1992-1994. INTA. Centros Regionales Misiones, Corrientes y Entre Ríos.

HAYWOOD, J.D., J.D. BURTON. 1990. Phosphorus fertilizer, soil, and site preparation influence loblolly pine productivity. New Forest 3: 275-287.

INTA. 1990. Atlas de suelos de la República Argentina. Castelar. Buenos Aires, Argentina.

NAMBIAR, S. 1998. Productivity and sustainability of platation forests. En: Actas X Silvotecnia. Conferencia IUFRO. Mejoramiento y productividad de sitios. Concepción, Chile, p. 278-308.
MASON, E. G. 1992, Decision support system for establishing Radiate pine plantation in the Central North Island of New Zealand, PhD Thesis, University of Canterbury, New Zealand, $301 \mathrm{p}$.

PRITCHETT, W.L. 1986. Suelos forestales: Propiedades, conservación y mejoramiento. México. Limusa, $634 \mathrm{p}$.

SNOWDON, P., H.D. WARING. 1984. Long-term nature of growth responses obtained to fertilizer and weed control applied at planting and their consequences for forest management. En: Proceedings Symposium on Site and Productivity of Fast Growing Plantations. IUFRO. Pretoria, South Africa. Volume 1: 701-711.

TORO, J. 1995. Avances en fertilización en Pinus radiata y Eucalyptus en Chile. En: Actas Simposio IUFRO. Manejo Nutritivo de Plantaciones Forestales: 293-299. Valdivia, Chile. 